\title{
Analysis of eigenfrequencies in piezoelectric transducers using the finite element method
}

Jensen, Henrik

Published in:

IEEE Ultrasonics Symposium Proceedings

Link to article, DOI:

10.1109/ULTSYM.1988.49405

Publication date:

1988

Document Version

Publisher's PDF, also known as Version of record

Link back to DTU Orbit

Citation (APA):

Jensen, H. (1988). Analysis of eigenfrequencies in piezoelectric transducers using the finite element method. In IEEE Ultrasonics Symposium Proceedings (Vol. Volume 1, pp. 393-396). IEEE.

https://doi.org/10.1109/ULTSYM.1988.49405

\section{General rights}

Copyright and moral rights for the publications made accessible in the public portal are retained by the authors and/or other copyright owners and it is a condition of accessing publications that users recognise and abide by the legal requirements associated with these rights.

- Users may download and print one copy of any publication from the public portal for the purpose of private study or research.

- You may not further distribute the material or use it for any profit-making activity or commercial gain

- You may freely distribute the URL identifying the publication in the public portal

If you believe that this document breaches copyright please contact us providing details, and we will remove access to the work immediately and investigate your claim. 


\section{ANALYSIS OF EIGENFREQUENCIES IN PIEZOELECTRIC TRANSDUCERS USING THE FINITE ELEARIT METHOD.}

HENRIK JENSEN

Institute of Manufacturing Engineering

Technical University of Denmark, DK-2800 Lyngby, Denmark.

\section{ABSTRACT}

Analysis of piezoelectric transducers is often based on one-dimensional methods such as equivalent circuits and transmission line models. This implies that the vibrational pattern is presumed rather than found by the analysis. The presumptions are valid only for simple geometries such as thin plates. The finite element method can determine the nature of the vibrations and is applicable to any geometry. Therefore it is a valuable supplement to the traditional methods for design of new transducer types. Computer programs for analysis of axisymmetric transducers, which include the complete set of piezoelectric equations have been developed. They can find eigenfrequencies for undamped transducers and make forced response analysis for transducers with internal and radiation damping. Super element technique is employed to model the transducer backing in an efficient way. Examples ranging from a freely vibrating disk to a real focused transducer with a conical backing are presented.

\section{INTRODUCTION.}

The finite element method (FEM) is a method for solving partial differential equations numerically. It can handle not oniy scalar but also vectorial problems. In solid mechanics it has become the method of choice. The method has also been used for piezoelectric materials for almost 20 years [1-3], but its use in ultrasonics is stil] limited. There are several reasons for this, and the most important may be that the one-dimensional methods such as Mason's equivalent circuits and the KLM model in many cases work quite well. It is also important that FEM is computationally demanding and that numerical methods often give less insight in the problem. Still FEM offers an opportunity to take more design parameters into account in the analysis than the traditional methods do. Examples may be the effect of curvature, partial electrode coverage, edge effects and varying cross section. As the analysis not necessarily involves much manual work if it is combined with appropriate pre- and post- processors there should be a place for FEM in the transducer designers toolbox.

A specialized computer program called Curie has been written [4] to utilize the potential of the method to calculations involving piezoelectric transducer. It is currently only intended for axi-symmetric transducers, but it includes the full set of piezoelectric equations, the influence of a fluid load, internal damping and makes use of super element technique. Some of these features are included as they are useful in the design process, others make the program more efficient. Furthermore the program is supported by a PC based preand post-processor.

\section{Finite Glenent Analysis.}

When a transducer is to be analyzed with FEM it must first be described geometrically in a coordinate system, $x_{i}$. The subscript $i$ and later $j, k$, and 1 may assume the values 1,2 and 3 corresponding to the three coordinate axes. The transducer geometry is defined by a number of elements. Each element is associated with a number of nodes with coordinates $x_{i m}$, where the second subscript $m=1,2, \ldots, M$. is the node number. The main assumption in the method is that the electric potential, $\varphi$ and the displacement, $u_{j}$ within the element can be determined with an acceptable accuracy by interpolation between their nodal values denoted by:

$$
\begin{aligned}
& u_{j}\left(x_{i m}\right)=u_{j m} \\
& \varphi\left(x_{i m}\right)=\varphi_{m}
\end{aligned}
$$

The interpolation is done in accórdance with the shape functions, $N_{m}$ which are characteristic for the Elements.

$$
\begin{aligned}
u^{*}\left(x_{i}\right) & =N_{m}\left(x_{i}\right) u_{j m}(2 . a) \\
\varphi^{\star}\left(x_{i}\right) & =N_{m}\left(x_{i}\right) \quad \varphi_{m}(2 . a)
\end{aligned}
$$

The asterisk is to distinguish the interpolated functions from the true functions.

The interpolated displacements are differentiated spatially to find the strain tensor, $\mathbf{s}_{\mathbf{i} j}$, 


$$
s_{i j}=\frac{1}{2}\left(u^{*}{ }_{i, j}+u^{*}{ }_{j, i}\right)(3 . a)
$$

and the electric field vector, $E_{i}$,

$$
E_{i}=\varphi^{*}, i \quad(3 . b)
$$

The constitutive equations are then used to find the stress tensor, $\mathrm{T}_{\mathrm{kl}}$, and the dielectric displacement, $D_{k}$.

$$
\begin{aligned}
& \mathrm{T}_{k l}=c_{i j k l} S_{i j}-e_{i k l} E_{i}(4 . a) \\
& D_{k}=e_{k i j} s_{i j}+\epsilon_{i k} E_{i}(4 . b)
\end{aligned}
$$

Here and throughout this paper is used the convention that repeated subscripts in a term imply a summation over all allowable values of the subscript for that term. once again the shape functions are used, but this time as weight functions to integrate the divergence of $T_{k l}$ and $D_{k}$ on the coordinate axes into equivalent nodal forces, $F_{k m}$, and equivalent nodal charge, $\mathrm{Q}_{\mathrm{m}}$.

$$
\begin{aligned}
F_{k m} & =\int_{V} N_{m}\left(x_{i}\right) T k l, 1 d V \quad(5 . a) \\
\mathrm{g}_{m} & =\int_{V} N_{m}\left(x_{i}\right) D_{1, l} d V \quad(5 . b)
\end{aligned}
$$

$\mathrm{V}$ is the volume of the element.

The nodal forces must satisfy the equation of motion and an electric charge may not be accumulated in the nodes, therefore:

$$
\begin{aligned}
F_{k m} & =M_{m n} \ddot{u}_{k n} & (6 . a) \\
\mathrm{Q}_{\mathrm{m}} & =0 & (6 . b)
\end{aligned}
$$

where $M_{m n}$ represents the mass of the structure in form of a mass matrix. The socalled consistent mass matrix, $\mathbf{M}^{1} \mathrm{mn}$, is calculated by integrating the mass density, $\rho$, with the shape functions as weight functions

$$
\mathrm{M}_{\mathrm{mn}}^{1}=\int_{\mathrm{V}} \rho \mathrm{N}_{\mathrm{m}}\left(\mathrm{x}_{i}\right) \mathrm{N}_{\mathrm{n}}\left(\mathrm{x}_{i}\right) \mathrm{dV}(7)
$$

Alternatively the mass matrix may be lumped ie. only have non-zero elements in the diagonal. In ultrasonic applications, where the wavelengths are small compared with typical dimensions, a linear combination of the two is superior with respect to convergence to the true phase velocities.

When the operations given by equation 2 through 7 are combined they provide a relation between the nodal displacements and the electric potential on one side and the nodal force and nodal charge on the other. As a result of how the shape functions are defined in FEM [5] the relation may be given in the form

\section{4 - 1988 ULTRASONICS SYMPOSIUM}

$\begin{aligned} F_{k m} & =D_{k l m n} u_{1 n}+D_{k 4 m n} \varphi_{n}(8 . a) \\ -Q_{m} & =D_{4 l m n} u_{l n}+D_{44 m n} \varphi_{n}(8 . b)\end{aligned}$

where $D_{k l m n}=\left(K_{k l m n}-\omega^{2} \delta_{k l} M_{m n}\right)$ is the dynamic stiffness matrix, and $\mathrm{K}_{\mathrm{k} / \mathrm{mn}}$ is the static stiffness matrix. $\delta_{k l}$ is Kronecker's delta. The subscript 4 is used to include the electrical degree of freedom in the same frame as the purely mechanical degrees of freedom. $D_{k 4 m n}$ and $D_{44} \mathrm{mn}$ are piezoelectric and electric generalized stiffness matrices, which have no term equivalent to the mass term.

In this form the method by analogy is equivalent to an electric L-C network, but as opposed to the common use of equivalent circuits, the method itself provides values for the components.

When the stiffness matrices for all the elements are calculated, they are assembled into a complete FEM model of the same form as equation ( 8 ). Boundary conditions are enforced directly on the nodes.

\section{Super Elements.}

The first and most important consideration using FEM is to find symmetries or periodicities that can reduce the size of the model. Most drastic reduction may be obtained if the dimensionality of the problem can be reduced, as in the case of axial symmetry. Similar reduction may be obtained by super element technique if a part of the transducer is cylindrical or periodic. This may often be the case with the backing.

A super element is an assemblage of ordinary elements which is treated as one single element. Internal node can be eliminated with no loss in accuracy for a single frequency, but the price in an increased bandwidth in the stiffness matrix. The advantages are that substructures may be analyzed independently and that super elements may be re-used several times in the same model.

A cylinder can be modelled by $2^{\mathrm{m}}$ identical layers. When all nodes except those on the two faces of the layer are eliminated the force displacement relation is most conveniently written in matrix form

$$
\left[\begin{array}{l}
F_{1} \\
F_{2}
\end{array}\right]=\left[\begin{array}{ll}
D_{11} & D_{12} \\
D_{21} & D_{22}
\end{array}\right]\left[\begin{array}{l}
u_{1} \\
u_{2}
\end{array}\right]
$$

$F$ and $u$ are vectors of generalized force and displacement degrees of freedom (DOFs). Subscript 1 and 2 refers to the two faces of the layer. By combining the two layers and eliminating the DOFs on their interface the super element for two layers becomes: 


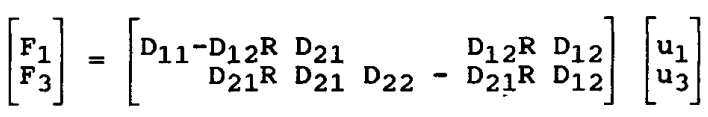

where $R=\left(D_{11}+D_{22}\right)^{-1}$ and subscript 3 refers to the second face of the second layer. The computational price of this doubling procedure is $\approx \mathbf{M}^{3}$, where $M$ is the number of DOFs on the faces of the layers. Hence the price of making a super element of $2^{\mathrm{m}}$ layers is $\approx \mathrm{mM}^{3}$ whereas the price of direct elimination would be $\approx\left(2^{\mathrm{m}}-1\right) \mathrm{M}^{3}$. The saving is similar to that of using the FFT algorithm instead of direct evaluation of the DFT.

\section{Element size.}

The next consideration is the size of the elements. The importance of it can be seen from the fact that the price of solving $M$ linear equations with at bandwidth in the coefficient matrix of $B$ is $\approx \mathrm{MB}^{2}$. Hence the price for solving a n-dimensional is inversely proportional to the element size in the $(3 n-2)$ th. power.

An acceptable element size may be estimated from error in the phase velocity introduced by the discretization. The phase velocity can be found by analyzing an infinite waveguide if finite sized layers [4]. Fig. 1 shows the normalized phase velocity, $c_{\varphi} / c_{0}$, vs. the normalized element size, $l / \lambda$, where $\lambda$ is the wave length. The phase velocity is shown for various mass matrices, $M^{\alpha}$, which are linear combinations of the consistent, and the lumped, $\mathrm{M}^{0}$, mass matrices. $\mathrm{M}^{\alpha}=\alpha \mathrm{M}^{1}+(1-\alpha) \mathrm{M}^{0}$.

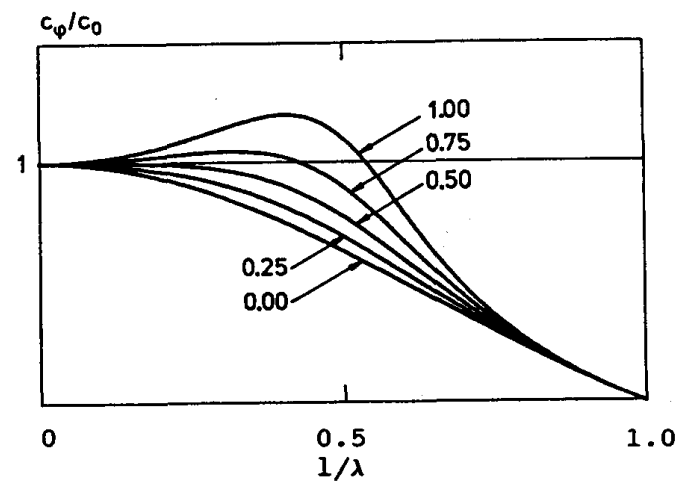

Fig. 1. Phase velocity vs. element size in FEM model for various mass matrices.

It can be seen that the lumped mass matrix results in too low a phase velocity whereas for $1 / \lambda<0.5$ the consistent mass matrix results in too high a phase velocity and that a combination in most cases is superior.

If the relative error of the phase velocity is required to be within a limit, $\pm \epsilon$, an optimal value, $\alpha_{\text {opt, }}$ can be chosen so $1 / \lambda$ becomes largest possible before the error is exceeded. Selected values can be seen from the table below.

\begin{tabular}{|c|c|c|}
\hline$\epsilon[q]$ & $\alpha_{\text {opt }}$ & $1 / \lambda<$ \\
\hline 0.1 & 0.54 & 0.20 \\
0.2 & 0.55 & 0.25 \\
0.5 & 0.58 & 0.29 \\
1.0 & 0.61 & 0.34 \\
2.0 & 0.66 & 0.40 \\
5.0 & 0.80 & 0.49 \\
\hline
\end{tabular}

Table 1. Optimal a values and allowable element size for selected acceptable errors in phase velocity.

\section{v. Eigenfrequency Analysis.}

The undamped eigenfrequencies of a transducer can be found from the FEM model by solving the algebraic eigenvalue problem that arise from the equations if $\omega$ is considered to be unknown. The model has as many eigenvalues as there are DOFs, but only very few of interest. Therefore the eigenvalues and eigenvectors are found by inverse sub-space iteration, as this method can be used to find a few eigenvectors in a selected frequency range via a frequency shift in the iterations. Even then the method may find many eigenfrequencies that are of little interest as their

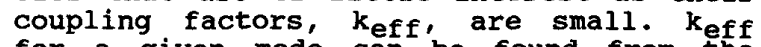
for a given mode can be found from the eigenfrequencies for short circuit $\left(f_{s}\right)$ and free electrodes $\left(f_{p}\right)$ [6]. This can be used to discriminate the modes with low coupling. Still their presence may degrade the convergence of the iterations and require more vectors to be iterated simultaneously.

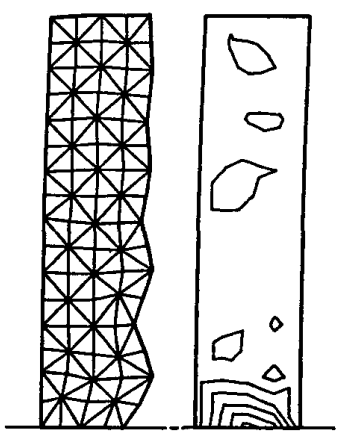

Short circuit $f_{\mathrm{s}}=1.945 \mathrm{MHz}$.

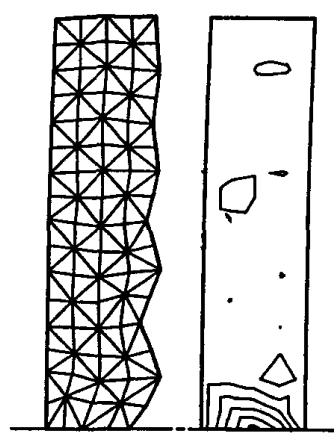

Disconnected $f_{p}=1.954 \mathrm{MHz}$.
Fig. 2. Mode of curved disk with constraint to prevent bending. 


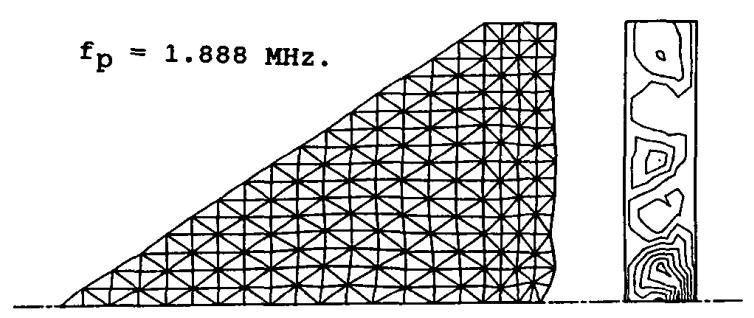

$\mathrm{f}_{\mathrm{S}}=1.880 \mathrm{MHz}$.

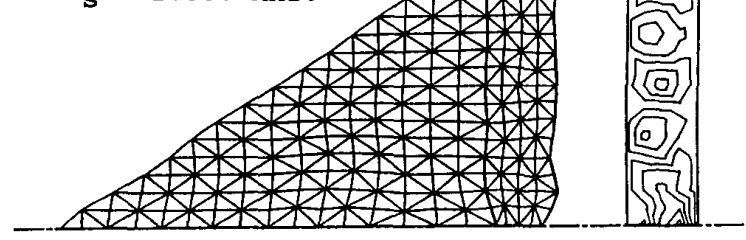

Fig. 3. Mode of transducer with conical backing for short circuit and free electrodes.

The boundary conditions or constraint equations may then be used to suppress un interesting modes. Fig. 2 shows an example of a freely vibrating spherical cap for a weakly focused transducer, where the bending modes have been suppressed by constraining the nodes on the back of the cap to have same axial displacement. Both the short circuit and the free electrode conditions are shown. The diameter is $8 \mathrm{~mm}$. and the thickness is $1 \mathrm{~mm}$. The material is PZ-29 from Ferroperm. This and the next figures show the deformed grid for the complete model and contours of constant electric potential for the piezoelectric part. The models are axi-symmetric around the horizontal baseline.

Figure 3 shows the same disk as figure 2 but now it has been mounted on a conical backing of cast iron, which has nearly the same impedance as the PZ-29.

\section{Steady State Solution.}

If radiation losses or internal damping is included in the analysis and finite valued boundary conditions are enforced the eigenfrequency analysis is no longer applicable. The equations may then be solved for a single frequency at a time as a set of linear equations. In combination with FFT this method can be used to find the transient response of a transducer. Figure 4 shows the transducer from figure 3 , but now radiating into water at the frequency of maximum radiated effect.

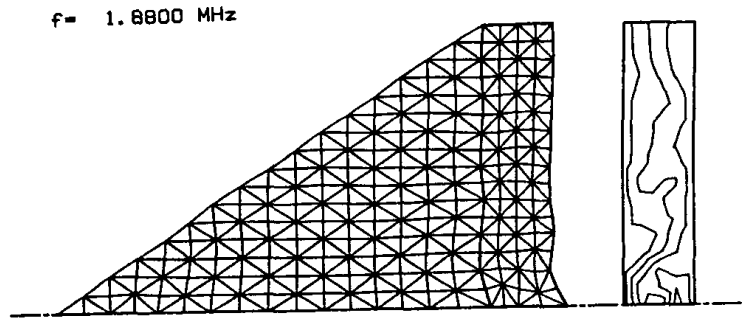

Fig. 4. Vibration pattern for transducer radiating into water at the frequency of maximum output effect.

VII. Conclusion

The finite element method is a standard method that is applicable to the analysis of piezoelectric transducers. The method can be computationally demanding, so care must be taken to keep the FEM models small. Some methods for doing this have been indicated and typical examples of the use of FEM have been given to demonstrate the kind of results that is obtained. To utilize the method, it is important to have good postprocessing facilities to generate graphs and compress the information in the results.

[1] ALLIK, H. and HUGHES, T.J.R. (1970). Finite Element method for Piezoelectric vibration, Int. J. Num. Meth. Eng. 2, 151-157.

[2] KAGAWA, $Y$. and YAMABUCHI, $T$. (1979). Finite Element Simulation of a Composite Piezoelectric Ultrasonic Transducer, IEEE Trans. Sonics Ultrason. SU-26, 81-88.

[3] DÉCARPIGNY, J.N. et. al. (1985) Inair analysis of piezoelectric Tonpilz transducers in a wide frequency band using a mixed finite element-plane wave method, J. Acoust. Soc. Am 78, 1499-1507.

[4] JENSEN, H. (1986). Calculations for Piezoelectric Ultrasonic Transducers, Risø-R-536, Risø National Laboratory, DK-4000 Roskilde, Denmark

[5] ZIENKIEWICZ, O.C. (1977) The Finite Element Method, 3. Ed. (McGrawHi11, London)

[6] LOCKE, S., KUNKEL, H.A. and PIKEROEN, B.' (1987) Finite Element Modelling of Piezoelectric Ceramic Disks, Proc. IEEE Ultrasonics Symp., 701-706.

\section{$396-1988$ ULTRASONICS SYMPOSIUM}

